\title{
Klein gleich groß?
}

Der ALLTAG IN EINER KINDERCHIRURGIE gleicht in vielerlei Hinsicht dem einer „normalen“ Chrirurgie - Stichwort Leistungssteigerung, Kostenreduktion, Personalengpässe. Und doch gibt es so viele Dinge, die anders sind: Lachende und weinende kleinen Patienten, besorgte und erleichterte Eltern, ein Clown, der ablenkt und zum Lachen bringt.

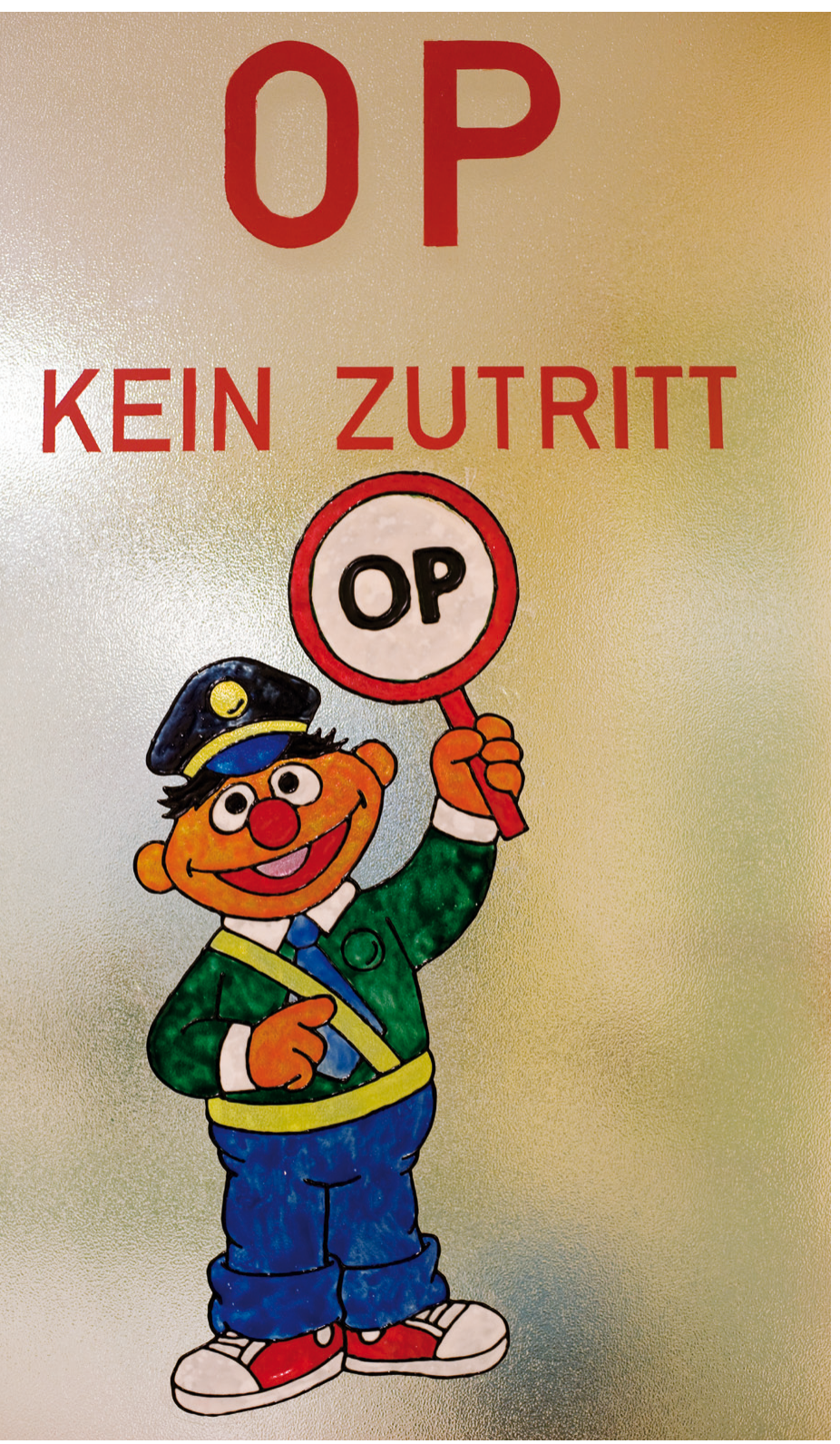

chirurgie und plastischen Korrekturen. Die wichtigste Voraussetzung, um hier zu arbeiten, ist ein großes $\mathrm{Ma}$ an Empathie für Kinder und vor allem für ihre Eltern. Für diese ist es unglaublich schwierig, ihre Kinder in fremde Hände zu geben, ohne dass sie Einfluss nehmen können auf das weitere Geschehen, wie es im OP der Fall ist. Viele bringen dieses Vertrauen nur mit größter Mühe auf. Umgekehrt ist auch für die Kinder das Loslösen von den Eltern, der Trennungsschmerz, die Begegnung mit völlig fremden und anders gekleideten Menschen eine völlig neue Erfahrung und eine große Stresssituation.

Darauf angemessen zu reagieren setzt ein sehr professionelles Verhalten aller Mitarbeiter voraus. Ein Chirurg der Abteilung hat es in einer Teambesprechung einmal ganz treffend formuliert: „Wenn Eltern sich im Ton vergreifen, ist das immer der Angst und der Sorge um ihr Kind geschuldet." Diese Einstellung ist sehr wichtig für den täglichen Betrieb.

Die Kinderklinik Amsterdamerstraße in Köln ist eine Klinik der Maximalversorgung mit vier Operationssälen und einer Endoskopie. Das operative Spektrum reicht von der Kinderchirurgie, Kinderurologie und Traumatologie bis zur Hand-
Das ideale Team: starke Eltern, kreative OP-Pflege

Damit eine Operation für die kleinen Patienten möglichst stressfrei verlaufen kann, leisten im Idealfall die Eltern einen großen Beitrag. Sie können mit altersgemäßen und anschaulichen Erklärungen ihr Kind sowohl auf den bevorstehenden Krankenhausaufenthalt als auch auf die anstehende Operation vorbereiten. $\mathrm{Zu}$ diesen Themen gibt es eine Vielfalt an Kinderbüchern $(\rightarrow$ Buchtipp). So lernen die Kinder die Abläufe bereits im Vorfeld kennen und kommen mit der ungewohnten Situation besser zurecht. Ganz wichtig ist, dass Eltern ihre eigenen Emotionen kontrollieren - insbesondere in der Zeit, in der sie ihre Kinder in den Aufwachraum begleiten. Notfalls sollten Sie sich für einige Zeit vom Kind abwenden, um ihm dann wieder emotional gestärkt den Eindruck von Sicherheit und Verlässlichkeit zu geben.

Für die OP-Pflege liegt die größte Herausforderung der Kinderchirurgie in der Kreativität. Sei es die Lagerung des Patienten, die Nutzung des vorhandenen Einmalmaterials und nicht zuletzt die Begleitung des Kindes bis in den Narkoseschlaf. Bereits an der OP-Schleuse, wo die Eltern ihre Kinder abgeben, ist es wichtig, einen vertrauensvollen Kontakt aufzubauen. Wie beim Erwachsenen stellt man sich dem Kind selbstverständlich ohne Mundschutz vor. Im Gegensatz zu einem Erwachsenen bemüht man sich spielerisch, ein Vertrauensverhältnis zu schaffen. Viele Kinder brauchen Körperkontakt, wollen in den Arm genommen werden, andere lassen sich beeindrucken, indem man die Vorbereitungen im OP als spannendes Spiel gestaltet, für das es anschließend auch eine Tapferkeitsurkunde gibt (über die Firmen Abbott oder Emla zu beziehen).

\section{Herausforderungen: kindgerechte} Lagerung, richtige Instrumentenwahl Der Erwachsene wird in der Regel mittels des Operationstisches und den Raffinessen, welcher diese mit sich bringt, gelagert. Bei einem Kind geht das nicht ohne weiteres. Hier sind Größe und Gewicht entscheidend. Handelt es sich um ein Frühgeborenes, muss die Lagerung sehr 
viel vorsichtiger durchgeführt werden als bei einem Säugling oder Kleinkind, denn die dünnen Knochen sind überaus empfindlich. Watte und kleine Gelpolster sind hier unverzichtbare Hilfsmittel.

Sehr wichtig ist, dass die Kinder nicht auskühlen. Der OP-Saal wird dazu immer auf eine ideale Temperatur beheizt, die abhängt vom Gewicht des Kinds und seiner aktuellen Körpertemperatur. Außerdem werden alle Kinder auf eine spezielle Wärmematte gelegt, bei der warme Luft durch eine Papierdecke direkt an den Patienten abgegeben wird. Da Kinder den größten Wärmeverlust über den Kopf erleiden (wegen der relativ großen Oberfläche), ist es ratsam, den Frühgeborenen kleine Mützen aus Watte zu basteln.

Für die HF-Chirurgie werden Kinderelektroden verwendet; bei Frühgeborenen koaguliert der Chirurg ausschließlich bipolar. Was das Instrumentarium angeht, hat sich der Markt in den letzten zwanzig Jahren deutlich erweitert. Behalf man sich früher beispielswiese mit Mikroinstrumentarium aus der Augenchirurgie, gibt es heute längst sehr viel Auswahl an speziellem Kinder-Instrumentarium für jeden Eingriff, beispielsweise aus besonders leichtem Titan. Die Wahl der Instrumente richtet sich auch nach Größe und Gewicht: Je kleiner der Patient bzw. je geringer das Gewicht, desto fragiler das Gewebe bzw. die Organe - und umso leichter und filigraner müssen die Instrumente sein.

Unsere Kinderchirurgie hält Instrumente für Patienten zwischen 300 Gramm bis 110 Kilogramm vor. Bei diesen Gewichts- und Größenunterschieden kommt es sehr es auf die Flexibilität jedes Mitar- beiters an, ungeachtet der Berufsgruppe. Von der diffizilen Operationsweise des Frühgeborenen bis zum Teenager muss alles abgedeckt werden. Die Narkose und der operative Eingriff selbst können bei ähnlichem Krankheitsbild völlig unterschiedlich aussehen. Das gilt auch für den persönliche Umgang mit dem Patienten: Einen Dreizehnzehnjährigen muss man natürlich anders ansprechen als einen Drei- oder Siebenjährigen.

Diese ständige Anpassungsleistung macht die Tätigkeit in einer Kinderchirurgie sehr anspruchsvoll und abwechslungsreich. Das Schöne: Die Arbeit ist mit viel Freude und Fröhlichkeit verbunden, denn Kinder sind sehr ehrliche und auch dankbare Patienten. Und das Lächeln eines Kindes wiegt so manche Überstunde auf.

\section{TIPPS}

... von Mitarbeitern allgemeinchirurgischer OPs zum Umgang mit Kindern

- Das zu operierende Kind in der OP-Besprechung am Tag vor der OP vorstellen.

- Am OP-Tag sollten Kinder als möglichst als letzte eingeschleust werden, weil dann schon Ruhe an der Schleuse herrscht.

- Kinder bekommen zur Erleichterung der Venenpunktion im Vorfeld „Zauberpflaster“ (EMLA-Pflaster) appliziert.

- Eltern dürfen ihr Kind bis zur OPSchleuse begleiten. Der Anästhesist holt das Kind selbst an der Schleuse ab - ohne Mundschutz.

\section{HINTERGRUND}

Die Kinderchirurgie ist der jüngste Zweig der allgemeinen Chirurgie. Die erste deutsche Kinderklinik wurde 1863 in Nürnberg eingerichtet. Zu einem eigenständigen Fach entwickelte sie sich jedoch erst nach dem zweiten Weltkrieg. Was den Umgang und die Rechte eines jungen Patienten im Krankenhaus betrifft, gibt es eine Richtlinie: die „Europäische Charta für die Rechte des Kindes im Krankenhaus" aus dem Jahre 1988. Sie fasst in zehn Punkten die Rechte des Kindes zusammen. Sie ist einzusehen beim „Aktionskomitee Kind im Krankenhaus Bundesverband e. V.“ unter www. akik-bundesverband.de
- Wenn die Personalsituation es erlaubt, kann sich auch eine Begleitperson in einer „Elternschleuse“ umziehen und das Kind in den OP begleiten. Sobald das Kind schläft, bringt ein Mitarbeiter die Begleitperson wieder zurück zur Schleuse.

- Kuscheltiere oder Schmusetücher dürfen mit in den OP, bis das Kind schläft.

- Kleinere Kinder werden aus dem Bett direkt auf den OP-Tisch gelegt. Größere finden es manchmal spannend oder sogar lustig, wenn sie wie Erwachsene eingeschleust werden.

\section{BUCHTIPPS}

Susa Hämmerle, Kyrima Trapp: Heut gehen wir ins Krankenhaus. Für Kinder ab zweieinhalb Jahren. Betz, Wien 2001, ISBN 321910942X, EUR [D] 10,95

Andrea Erne, Marion Kreimeyer-Visse: Was passiert im Krankenhaus? Für Kinder zwischen 4 und 6 Jahren. Ravensburger, ISBN 3473328480, EUR [D] 12,95
- Alle Kinder sofort mit einem warmen Tuch zudecken; Babys sofort in den warmen OP bringen.

- Der Saal sollte gut vorgewärmt sein. Während der Einschlafphase sollte Ruhe herrschen (keine Container oder Einmalsachen öffnen, das Reden auf Flüsterlautstärke reduzieren).

- Die Eltern warten während der OP-Zeit vor dem OP und werden sofort zu ihren Kindern geführt, wenn diese in den Aufwachraum oder auf die Intensivstation kommen. Wenn die Kinder aufwachen, sind Eltern und Kuscheltier schon da.

AUTORIN
Iris Steugck
Leitende OP-Schwester
Kinderklinik Köln-Riehl
E-Mail: steugcki@kliniken-koeln.de

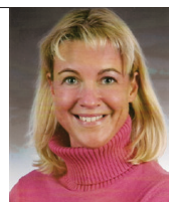

\section{BIBLIOGRAFIE}

DOI 10.1055/s-0032-1304794

Im OP 2012; 2: 66-67

(c) Georg Thieme Verlag KG

Stuttgart · New York · ISSN 1611-7905 